\title{
Percepção dos profissionais de saúde sobre condições de trabalho durante o enfretamento da COVID-19 nas maternidades públicas de Aracaju/SE
}

\author{
Perception of healthcare professionals about working conditions during the confronting COVID-19 \\ in public maternities in Aracaju/SE \\ Percepción de los profesionales de la salud sobre las condiciones de trabajo durante el \\ enfrentamiento al COVID-19 en maternidades públicas en Aracaju/SE
}

\author{
Derijulie Siqueira de Sousa \\ ORCID: https://orcid.org/0000-0001-7209-1034 \\ Universidade Tiradentes, Brasil \\ E-mail: deriartur@gmail.com \\ Andreia Centenaro Vaez \\ ORCID: https://orcid.org/0000-0002-1503-0785 \\ Universidade Federal de Sergipe, Brasil \\ E-mail: andreiacentenarovaez@gmail.com \\ Thialla Andrade Carvalho \\ ORCID: https://orcid.org/0000-0002-3301-5060 \\ Instituto Federal de Sergipe, Brasil \\ E-mail: thiallaba@hotmail.com \\ Carla Viviane Freitas de Jesus \\ ORCID: https://orcid.org/0000-0002-7775-6610 \\ Universidade Tiradentes, Brasil \\ E-mail: carlavfj@gmail.com \\ Íkaro Daniel de Carvalho Barreto \\ ORCID: https://orcid.org/0000-0001-7253-806X \\ Universidade Federal de Pernambuco, Brasil \\ E-mail: daniel.carvalho.ib@gmail.com \\ Margarete Zanardo Gomes \\ ORCID: https://orcid.org/0000-0003-2395-5523 \\ Universidade Tiradentes, Brasil \\ E-mail: margarete_zanardo @itp.org.br \\ Francisco Prado Reis \\ ORCID: https://orcid.org/0000-0002-7776-1831 \\ Universidade Tiradentes, Brasil \\ E-mail: franciscopradoreis@gmail.com
}

\begin{abstract}
Resumo
A pandemia do Coronavírus (COVID-19) atingiu os serviços de saúde e gerou uma demanda extra de estruturas, insumos e recursos humanos, o que desafiou os sistemas de saúde, inclusive as maternidades, que não suspenderam suas atividades. Diante da realidade específica das maternidades públicas, o estudo teve como objetivo descrever a percepção das equipes de saúde sobre o ambiente de trabalho risco da COVID- 19. Trata-se de um estudo descritivo com abordagem quantitativa, realizado com profissionais de saúde que atuavam na assistência materno-infantil em maternidades públicas do município de Aracaju/SE. A amostra foi realizada por conveniência e contou com a participação de 218 profissionais de saúde que atuam nessas maternidades. Os dados apontaram que, no geral, as condições de biossegurança quanto à equipamentos e capacitação dos profissionais para seu uso não foram adequadas e seguras. Por outro lado, vale destacar que os profissionais e seus colegas não se sentiram capacitados e emocionalmente seguros para o enfretamento da pandemia. Por fim os participantes do estudo referiram que as estratégias de enfrentamento foram incipientes, que a pandemia influenciou completamente a carga horária de trabalho, mas não interferiu na produção do trabalho.
\end{abstract}

Palavras-chave: Condições de trabalho; Infecções por coronavírus; Equipamentos de Proteção; Saúde do trabalhador.

\section{Abstract}

The Coronavirus (COVID-19) pandemic hit health services and generated an extra demand for structures, inputs and human resources, which challenged health systems, including maternity hospitals, which did not suspend their activities. Given the specific reality of public maternity hospitals, the study aimed to describe the perception of health teams about the risk work environment of COVID-19. This is a descriptive study with a quantitative approach, carried out with health professionals who worked in the maternal and childcare in public maternity hospitals in the city of 
Aracaju/SE. The sample was carried out for convenience and included the participation of 218 health professionals who work in these maternity hospitals. The data showed that, in general, the biosafety conditions regarding equipment and training of professionals for its use were not adequate and safe. On the other hand, it is worth noting that professionals and their colleagues did not feel empowered and emotionally safe to face the pandemic. Finally, the study participants reported that the coping strategies were incipient, that the pandemic completely influenced the workload, but did not interfere with the production of work.

Keywords: Working conditions; Coronavirus infections; Personal protective equipment; Occupational health.

\section{Resumen}

La pandemia del Coronavirus (COVID-19) golpeó los servicios de salud y generó una demanda extra de estructuras, insumos y recursos humanos, lo que desafió a los sistemas de salud, incluyendo las maternidades, que no suspendieron sus actividades. Dada la realidad específica de las maternidades públicas, el estudio tuvo como objetivo describir la percepción de los equipos de salud sobre el ambiente de trabajo de riesgo de la COVID-19. Se trata de un estudio descriptivo con abordaje cuantitativo, realizado con profesionales de la salud que actuaban en el área maternoinfantil. atención al niño en maternidades públicas de la ciudad de Aracaju/SE. La muestra se realizó por conveniencia y contó con la participación de 218 profesionales de la salud que laboran en estas maternidades. Los datos mostraron que, en general, las condiciones de bioseguridad en cuanto a los equipos y la formación de los profesionales para su uso no eran adecuadas y seguras. Por otro lado, cabe señalar que los profesionales y sus colegas no se sintieron empoderados y emocionalmente seguros para enfrentar la pandemia. Finalmente, los participantes del estudio relataron que las estrategias de enfrentamiento eran incipientes, que la pandemia influyó completamente en la carga de trabajo, pero no interfirió en la producción de trabajo.

Palabras clave: Condiciones de trabajo; Infecciones por Coronavirus; Equipo de protección personal; Salud laboral.

\section{Introdução}

A doença do coronavírus 2019 (COVID-19), doença provocada pelo SARS-CoV-2, provocou uma pandemia que atingiu os serviços de saúde gerando uma demanda extra de estruturas, insumos e recursos humanos, o que desafiou os sistemas de saúde de diversos países. O Brasil, devido a sua heterogeneidade regional, tem enfrentado grandes adversidades no enfrentamento da pandemia (Koh, 2020; Rache et al., 2020).

A pandemia mostrou as fragilidades existentes nos diferentes setores da saúde, inclusive, na garantia a segurança dos profissionais de saúde. Os trabalhadores de saúde envolvidos direta e indiretamente no enfrentamento à pandemia, são submetidos a várias formas de exposição (Ferioli et al., 2020; Oliveira et al., 2021).

$\mathrm{O}$ acesso a equipamentos de proteção individual (EPI) para profissionais de saúde é uma preocupação constante. A escassez de EPIs foi observada em diversas instituições brasileiras. Devido a essa escassez, os profissionais de saúde recorreram até à reutilização de equipamentos de uso único ou desenvolveram soluções rápidas genéricas locais, dos quais não oferecem a mesma proteção (Buckley et al., 2020; Carvalho et al., 2020).

Em Wuhan, o epicentro do surto, os profissionais médicos relataram o uso de fita adesiva para consertar máscaras rasgadas, a reutilização dos óculos de proteção e o embrulhe os dedos dos pés em sacos plásticos, por falta de disponibilidade de capas para calçados (Nagesh et al., 2020; Medeiros, 2020).

De acordo com os resultados da pesquisa Condições de Trabalho dos Profissionais de Saúde no Contexto da COVID19, realizada pela Fiocruz, 43,2\% dos profissionais de saúde sentiram-se desprotegidos no trabalho durante o enfrentamento da COVID-19, e o principal motivo, foi relacionado à falta, escassez e à inadequação do uso de EPIs, 64\% dos profissionais revelaram a necessidade de improvisar equipamentos. Os participantes da pesquisa também relataram o medo de se contaminar no trabalho (18\%), a ausência de estrutura adequada para realização da atividade (15\%), além de fluxos de internação ineficientes (12,3\%). O despreparo técnico dos profissionais para atuar na pandemia foi citado por 11,8\%, enquanto $10,4 \%$ denunciaram a insensibilidade de gestores para suas necessidades profissionais (Tavares, 2020).

Compreende-se por condições de trabalho as situações relativas aos meios físicos e materiais para a realização das atividades laborais, como os insumos e equipamentos. Desse modo, é por meio das condições de trabalho que são analisadas as inserções no mercado de trabalho: contratações, estabilidade na carreira, bem como riscos à saúde e à segurança no cotidiano profissional. (Leao et al., 2012) 
Para Huh (2020), os profissionais de saúde passaram então a enfrentar um recrudescimento da carga de trabalho, associado à possibilidade de serem expostos e infectados pelo SARS-CoV-2. O autor afirmou que a segurança dos profissionais de saúde é imprescindível para que eles ofereçam a melhor assistência para as pessoas infectadas. Ele relatou ainda que a sobrecarga de trabalho foi decorrente da escassez de médicos e de outros profissionais de saúde para cuidar do grande volume de pacientes.

A garantia da segurança e proteção efetiva dos trabalhadores da saúde, é um dos grandes desafios enfrentados nessa pandemia, particularmente nas maternidades, onde os serviços não puderam ser suspensos e que a estrutura física particular que recomenda leitos coletivos, não dispõe de ambientes adequados para isolamento respiratório. Diante da realidade específica das maternidades públicas, o estudo teve como objetivo descrever a percepção das equipes de saúde sobre o ambiente de trabalho risco da COVID- 19.

\section{Metodologia}

Trata-se de um estudo descritivo com abordagem quantitativa, realizado com profissionais de saúde que atuam na assistência materno- infantil em maternidades públicas do município de Aracaju/SE.

A amostragem foi efetuada por conveniência, as equipes das maternidades foram convidadas a participar do estudo através do preenchimento dos formulários online pela plataforma google forms. O link dos formulários foi compartilhado entre os integrantes da equipe pelos gerentes das unidades por meio de e-mail e do aplicativo de mensagens instantâneas (Whatzapp).

Embora a aplicação de questionário online tenha limitações metodológicas, ele foi uma escolha estratégica na pandemia, visto que durante o período da coleta de dados (entre setembro 2020 e março de 2021) Aracaju estava vivenciando um momento epidemiológico que recomendava o distanciamento social e limitação do número de pessoas circulando nas maternidades.

Foram incluídos na pesquisa todos os profissionais que trabalhavam nas instituições com vínculo formal há mais seis meses, e atuavam diretamente nos cuidados dos pacientes. Foram excluídos os profissionais que estavam de licença maternidade, férias ou atestado médico durante o período da coleta de dados.

Foram utilizados dois instrumentos de coleta de dados de autopreenchimento: um questionário sociodemográfico, e o segundo sobre percepção da equipe multiprofissional das maternidades no enfrentamento da COVID-19.

As variáveis categóricas foram descritas por meio de frequência absoluta e relativa percentual. As variáveis contínuas foram descritas por meio de média e desvio padrão. A hipótese de independência entre variáveis categóricas foi testada por meio dos testes Qui-Quadrado de Pearson ou Exato de Fisher. A hipótese de igualdade de médias foi testada por meio do teste T para amostras independentes. Foram estimadas razões de chances brutas e ajustadas por meio de regressão logística múltipla. O software utilizado foi o R Core Team 2021 (Versão 4.1.0) e o nível de significância adotado foi de 5\%.

O projeto seguiu as recomendações da resolução 466/2012/CSN/MS/CONEP, e foi aprovado pelo Comitê de Ética e Pesquisa (CEP) da Universidade Tiradentes (UNIT), em agosto do ano 2020, com o número de CAAE 35547720.7.0000.5371.

\section{Resultados}

Por meio do preenchimento de formulário online participaram do estudo 218 indivíduos da equipe multiprofissional, $53,7 \%$ (117), de uma maternidade de alto risco e 46,3\% (101) e de outra maternidade de risco habitual. Predominou em ambas as maternidades, profissionais do sexo feminino e a média de idade dos participantes foi de 39,2 anos. A Tabela 1 apresenta a composição das equipes de profissionais atuantes nas maternidades públicas de Aracaju durante a pandemia da COVID-19. 
Tabela 1: Composição das equipes de profissionais atuantes nas maternidades públicas de Aracaju durante a pandemia da COVID-19 - setembro/2020 a março/2021.

\begin{tabular}{|c|c|c|}
\hline & $\mathbf{n}$ & $\%$ \\
\hline \multicolumn{3}{|l|}{ Maternidades } \\
\hline Alto risco & 117 & 53,7 \\
\hline Risco habitual & 101 & 46,3 \\
\hline \multicolumn{3}{|l|}{ Sexo } \\
\hline Feminino & 201 & 92,2 \\
\hline Masculino & 17 & 7,8 \\
\hline \multicolumn{3}{|l|}{ Estado civil } \\
\hline Casado & 87 & 39,9 \\
\hline Solteiro & 84 & 38,5 \\
\hline União Estável & 17 & 7,8 \\
\hline Separado(a)/Divorciado(a) & 28 & 12,8 \\
\hline Viúvo(a) & 2 & 0,9 \\
\hline \multicolumn{3}{|l|}{ Possui filhos? } \\
\hline Sim & 143 & 65,9 \\
\hline Não & 74 & 34,1 \\
\hline \multicolumn{3}{|l|}{ Tem casa própria? } \\
\hline Sim & 156 & 71,9 \\
\hline Não & 61 & 28,1 \\
\hline \multicolumn{3}{|l|}{ Escolaridade } \\
\hline Ensino técnico & 56 & 25,7 \\
\hline Graduação & 47 & 21,6 \\
\hline Especialização/Residência & 97 & 44,5 \\
\hline Mestrado & 17 & 7,8 \\
\hline Doutorado & 1 & 0,5 \\
\hline
\end{tabular}

Legenda: $\mathrm{n}$ - frequência absoluta, \% - frequência relativa percentual. Fonte: Autores.

A Tabela 2, mostra dados quanto a formação profissional e das condições laboral dos participantes do estudo. Pode ser observado, com destaque, a ocorrência de uma participação expressiva dos componentes da equipe de enfermagem (enfermeiros e técnicos), os quais tinham do que 10 anos de atuação profissional; trabalhavam em sistema de plantão; tinham grande carga horária de trabalho; baixa remuneração; e que mais de $56 \%$ possuíam mais de um vínculo empregatício. Questionados quanto à satisfação com a profissão, 77,5\%, afirmaram estar satisfeitos, porém, e ainda, 46,5\% relataram que a expectativa de sua atuação profissional foi a esperada. 
Tabela 2: Formação profissional e condições laboral dos componentes das equipes de saúde que atuavam nas maternidades públicas de Aracaju/SE durante o enfrentamento da COVID-19.

\begin{tabular}{|c|c|c|}
\hline & $\mathbf{n}$ & $\%$ \\
\hline \multicolumn{3}{|l|}{ Profissão } \\
\hline Médicos & 35 & 16,05 \\
\hline Enfermeiros & 71 & 32,56 \\
\hline Fisioterapeutas & 9 & 4,12 \\
\hline Psicólogos & 5 & 2,29 \\
\hline Técnicos de enfermagem & 75 & 34,40 \\
\hline Outros & 23 & 10,55 \\
\hline $\begin{array}{l}\text { Tempo de atuação profissional } \\
\text { Menos de } 1 \text { ano }\end{array}$ & 11 & 5,1 \\
\hline 1 a 5 anos & 51 & 23,5 \\
\hline 6 a 10 anos & 43 & 19,8 \\
\hline Mais de 10 anos & 112 & 51,6 \\
\hline \multicolumn{3}{|l|}{ Setor de trabalho } \\
\hline Admissão & 18 & 8,7 \\
\hline Unidade de Internamento & 28 & 13,5 \\
\hline Unidade de Cuidados Neonatais & 82 & 39,6 \\
\hline Centro Obstétrico & 60 & 29,0 \\
\hline Outros & 19 & 9,2 \\
\hline \multicolumn{3}{|l|}{ Tempo de atuação no setor } \\
\hline Menos de 1 ano & 46 & 21,4 \\
\hline 1 a 5 anos & 67 & 31,2 \\
\hline 6 a 10 anos & 53 & 24,7 \\
\hline Mais de 10 anos & 49 & 22,8 \\
\hline \multicolumn{3}{|c|}{ Possui mais de um vínculo empregatício } \\
\hline $\operatorname{Sim}$ & 121 & 56,0 \\
\hline Não & 95 & 44,0 \\
\hline \multicolumn{3}{|l|}{ Trabalha em sistema de plantão? } \\
\hline Sim & 150 & 69,1 \\
\hline Não & 67 & 30,9 \\
\hline \multicolumn{3}{|c|}{ Está satisfeito com a sua atuação profissional? } \\
\hline $\operatorname{Sim}$ & 169 & 77,5 \\
\hline Não & 49 & 22,5 \\
\hline \multicolumn{3}{|c|}{ Que expectativa tem com relação a sua atuação profissional? } \\
\hline É o que eu esperava & 101 & 46,5 \\
\hline É menor do que esperava & 92 & 42,4 \\
\hline É maior do que eu esperava & 24 & 11,1 \\
\hline \multicolumn{3}{|c|}{ Quantas horas semanais dedica ao seu trabalho? } \\
\hline 30 horas & 61 & 28,0 \\
\hline 40 horas & 61 & 28,0 \\
\hline 50 horas & 23 & 10,6 \\
\hline Mais de 50 horas & 73 & 33,5 \\
\hline \multicolumn{3}{|c|}{ Renda pessoal (em salários-mínimos) } \\
\hline 1 a 3 salários-mínimos & 136 & 62,4 \\
\hline 4 a 6 salários-mínimos & 39 & 17,9 \\
\hline Mais que 6 salários-mínimos & 43 & 19,7 \\
\hline
\end{tabular}

Legenda: $\mathrm{n}$ - frequência absoluta. \% - frequência relativa percentual. Fonte: Autores.

$\mathrm{Na}$ Tabela 2, pode ainda ser observado que quanto a distribuição dos profissionais nos setores de trabalho das maternidades, a maior parte atuava em ambientes considerados fechados (centro obstétrico $29 \%$ e unidades de cuidados neonatais $39,6 \%$ ) e $31,2 \%$ deles, afirmaram que atuavam nesses setores, a cerca de 1 a 5 anos.

A Tabela 3 apresenta dados quanto a percepção das equipes de saúde que atuavam nas maternidades do estudo, em relação às condições de biossegurança no trabalho durante o enfrentamento da COVID-19. Os dados apontam, de acordo com as taxas percentuais encontradas que, no geral, as condições de biossegurança desde equipamentos e capacitação dos profissionais para seu uso não foram adequadas e seguras. Por outro lado, vale ainda destacar que os profissionais e seus 
colegas não se sentiram capacitados e emocionalmente seguros para o enfretamento da pandemia. Por fim a pandemia influenciou completamente a carga horária de trabalho e não interferiu na produção do trabalho e que mais da metade dos componentes das equipes estiveram ausentes de suas atividades laborais por motivo de saúde.

Tabela 3: Percepção das equipes de saúde quanto às condições de biossegurança nas maternidades do estudo durante o enfrentamento da COVID-19.

\begin{tabular}{|c|c|c|c|}
\hline & $\begin{array}{l}\text { Maternidade } \\
\text { Alto risco }\end{array}$ & $\begin{array}{c}\text { Maternidade } \\
\text { Risco habitual }\end{array}$ & p-valor \\
\hline Dispõe de EPIs adequados & $\mathrm{n}(\%)$ & $\mathrm{n}(\%)$ & \\
\hline Completamente & $31(26,5)$ & $63(62,4)$ & $<0,001^{*}$ \\
\hline Não completamente & $51(43,6)$ & $24(23,8)$ & \\
\hline Não & $35(29,9)$ & $14(13,9)$ & \\
\hline \multicolumn{4}{|c|}{ Dispõe de equipamentos adequados } \\
\hline Completamente & $23(19,8)$ & $54(53,5)$ & $<0,001 *$ \\
\hline Não completamente & $49(42,2)$ & $27(26,7)$ & \\
\hline Não & $44(37,9)$ & $20(19,8)$ & \\
\hline \multicolumn{4}{|c|}{ Dispõe infraestrutura adequada } \\
\hline Completamente & $10(8,5)$ & $23(23)$ & $0,001 *$ \\
\hline Não completamente & $19(16,2)$ & $26(26)$ & \\
\hline Não & $88(75,2)$ & $51(51)$ & \\
\hline \multicolumn{4}{|c|}{ Está seguro durante a utilização dos EPIs } \\
\hline Completamente & $20(17,1)$ & $50(49,5)$ & $<0,001 *$ \\
\hline Não completamente & $40(34,2)$ & $24(23,8)$ & \\
\hline Não & $57(48,7)$ & $27(26,7)$ & \\
\hline \multicolumn{4}{|c|}{ A pandemia influenciou sua carga horária de trabalho } \\
\hline Completamente & $64(55,2)$ & $47(47)$ & 0,447 \\
\hline Não completamente & $26(22,4)$ & $24(24)$ & \\
\hline Não & $26(22,4)$ & $29(29,0)$ & \\
\hline \multicolumn{4}{|c|}{ Os colegas de equipe estavam capacitados para o exercício do trabalho? } \\
\hline Completamente & $11(9,4)$ & $21(21)$ & $0,027 *$ \\
\hline Não completamente & $40(34,2)$ & $37(37)$ & \\
\hline Não & $66(56,4)$ & $42(42)$ & \\
\hline \multicolumn{4}{|c|}{ Sentiu- se capacitado para o enfrentar a pandemia } \\
\hline Concordo totalmente & $16(13,7)$ & $37(37)$ & $0,001 *$ \\
\hline Concordo mais que discordo & $50(42,7)$ & $33(33)$ & \\
\hline Não & $51(43,6)$ & $30(30)$ & \\
\hline \multicolumn{4}{|c|}{ A pandemia interferiu na sua produção de trabalho. } \\
\hline Completamente & $22(19)$ & $16(15,8)$ & 0,784 \\
\hline Não completamente & $37(31,9)$ & $31(30,7)$ & \\
\hline Não & $57(49,1)$ & $54(53,5)$ & \\
\hline \multicolumn{4}{|c|}{ Estava emocionalmente preparado para enfrentar a pandemia } \\
\hline Completamente & $17(14,5)$ & $30(30)$ & $0,016^{*}$ \\
\hline Não completamente & $42(35,9)$ & $34(34)$ & \\
\hline Não & $58(49,6)$ & $36(36)$ & \\
\hline \multicolumn{4}{|c|}{ Durante a pandemia, precisou ausentar-se por motivos de saúde? } \\
\hline $\operatorname{Sim}$ & $82(70,7)$ & $57(56,4)$ & $0,034 *$ \\
\hline Não & $34(29,3)$ & $44(43,6)$ & \\
\hline
\end{tabular}

Legenda: $\mathrm{n}$ - frequência absoluta. \% - frequência relativa percentual. Q - Teste Qui-Quadrado de Pearson. F - Teste Exato de Fisher. * $\mathrm{p}<0,05$. Fonte: Autores.

\section{Discussão}

As maternidades em face da pandemia, não suspenderam suas atividades e os profissionais que atuavam nesse ambiente tiveram que prestar cuidados sob uma forte pressão, em condições desfavoráveis e com interações emocionalmente exigentes (Ramaci et al., 2020; Costa et al., 2021). Esses profissionais tinham uma heterogeneidade de características sendo determinado por diferentes formas de exposição e ambientes de trabalho. Não foi diferente no presente estudo. Eram 
profissionais predominantemente do sexo feminino, casados ou em união estável, que tinham filhos e casa própria. Kang (2020) e Oliveira et al. (2020), relatam que entre os profissionais de saúde que estavam atuando na linha de frente foi percebido que além do receio do próprio contágio, eles temiam a infecção à sua família, colegas de trabalho e demais amigos, sentindo incertezas e rotulações e relutavam em trabalhar.

Um fator considerado, foi a carga de trabalho dos profissionais de saúde. No presente trabalho foi encontrado que mais da metade dos profissionais atuavam em sistema de plantão e possuíam altas jornadas de trabalho (mais de 50 horas semanais). Para Teixeira et al. (2020), a proteção pessoal dos profissionais de saúde é essencial para evitar a transmissão da COVID-19 e garantir segurança pessoal nos estabelecimentos de saúde. Além disso, problemas como cansaço físico, estresse, negligência ou insuficiência de medidas de proteção à saúde desses profissionais, não atingem igualmente as diferentes categorias e que a proteção pessoal dos profissionais de saúde é essencial para evitar a transmissão da COVID-19 e garantir segurança pessoal nos estabelecimentos de saúde (Mattos et al., 2022).

Em razão, possivelmente, da excessiva jornada de trabalho e baixa remuneração e apesar dos anos de atuação profissional, como também, dos anos de trabalho nos setores das maternidades em que atuavam, é que foi encontrado um significante sentimento de insegurança técnico e emocional, desses profissionais durante o enfrentamento da pandemia.

Para o enfrentamento dessa pandemia medidas preventivas precisaram ser implantadas, tais como, realização de atividades de educação permanente, atualização dos protocolos de biossegurança, maior aproveitamento de equipamentos e tecnologias, adoção de pausas esporádicas durante a jornada de trabalho, atenção ao clima organizacional para uma boa governança dos conflitos de tal forma a garantir ambientes de trabalho geradores de saúde física e mental (Barreto et al., 2020; Prigol et al., 2020).

A maioria dos profissionais das duas maternidades pesquisadas informaram que suas unidades de trabalho não forneciam estrutura adequada para o enfrentamento da pandemia. Para Fallucchi et al., (2021), a COVID-19 pode causar dano suficiente para sobrecarregar a infraestrutura de serviços de saúde, criando demandas extraordinárias e sustentadas nos sistemas de saúde e nos prestadores de serviços, inclusive nas maternidades que na sua rotina não incluíam a necessidade de leitos de isolamento respiratório para suspeitos e/ ou confirmados.

A garantia da segurança e proteção efetiva dos trabalhadores da saúde, tem sido um dos grandes desafios enfrentados nessa pandemia, considerando-se a alta transmissibilidade e velocidade de disseminação da COVID-19 (Adalja et al., 2020; Barreto et al., 2020). No atual estudo, foi encontrado que mais da metade dos profissionais que atuavam nas duas maternidades pesquisadas, durante o enfrentamento da pandemia, por motivos de saúde, tiveram de ser afastados de suas atividades laborais.

\section{Conclusão}

A atividade de cuidado é fundamental para a produção de saúde e bem-estar de toda uma sociedade. A atuação dos profissionais de saúde, inclusive nas maternidades, é capaz de proporcionar prevenção, promoção e recuperação em saúde. No entanto, o trabalho em saúde não está dissociado das contradições, em determinados ambientes, o (a) trabalhador (a), e expõe em risco a sua vida. Com isso, o que o cenário pandêmico tem evidenciado é a necessidade primordial do cuidado e da proteção daqueles (as) que estão à frente do enfretamento da pandemia da COVID-19.

Os participantes do estudo, que estavam atuando na linha de frente no combate à pandemia nas maternidades, referem que as estratégias de enfrentamento eram incipientes, o que aumentou o risco de adoecimento desses profissionais. A falta de Equipamentos de Proteção Individual (EPI), infraestrutura adequada, carga horária exaustiva, a insegurança sofrida pelos profissionais entre outros fatores, favoreceram à contaminação pela COVID-19 e exposição a outros problemas de saúde. Desta forma, recomenda-se que sejam realizados novos estudos que avaliem a promoção da saúde nos profissionais de saúde, no período pós-pandemia. 


\section{Referências}

Adalja, A. A., Toner, E., Inglesby, T. V. (2020). Prioridades para a comunidade de saúde dos EUA em resposta ao COVID-19. JAMA. 323(14):1343-1344

Barreto, M. L., Barros, A. J. D. D., Carvalho, M. S., Codeço, C. T., Hallal, P. R. C., Medronho, R. D. A., ... \& Werneck, G. L. (2020). O que é urgente e necessário para subsidiar as políticas de enfrentamento da pandemia de COVID-19 no Brasil?. Revista Brasileira de Epidemiologia, 23.

Buckley, C., Wee, S. L., \& Qin, A. (2020). China's doctors, fighting the coronavirus, beg for masks. The New York Times.

Carvalho, A. L., Assad, S. G. B., dos Santos, S. C. P., Rodrigues, G. V. B., Valente, G. S. C., \& Cortez, E. A. (2020). Atuação profissional frente à pandemia de COVID-19: dificuldades e possibilidades. Research, Society and Development, 9(9), e830998025-e830998025.

Costa, T. P., da Silva Ferreira, E., Rodrigues, D. P., Neri, D. T., Soares, E. A., de Freitas Ferreira, G. C., \& de Araújo, R. M. (2021). Os desafios da enfermagem obstétrica no início da pandemia da COVID-19 no Estado do Pará. Research, Society and Development, $10(3)$, e9510313042-e9510313042.

Fallucchi, F., Faravelli, M., Quercia, S. (2021). Alocação justa de recursos médicos escassos em tempos de COVID-19: o que as pessoas pensam?

Ferioli, M., Cisternino, C., Leo, V., Pisani, L., Palange, P., \& Nava, S. (2020). Protecting healthcare workers from SARS-CoV-2 infection: practical indications. European Respiratory Review, 29(155).

Huh, S. (2020). How to train health personnel to protect themselves from SARS-CoV-2 (novel coronavirus) infection when caring for a patient or suspected case. Journal of educational evaluation for health professions, 17.

Oliveira, A. M. C., da Silva Sousa, E., \& da Rocha Filho, D. R. (2021). Alterações físicas, emocionais e psicossociais de idoso na pandemia por coronavírus. Research, Society and Development, 10(6), e44310615964-e44310615964.

Oliveira, E. N., Costa, M. S. A., San Rodrigues, C., de Andrade, C. S. G., Mendonça, J. M. F., Pinto, M. R., ... \& Lima, G. F. (2020). Com a palavra os profissionais de saúde na linha de frente do combate à COVID-19. Research, Society and Development, 9(8), e30985145-e30985145.

Kang, L., Li, Y., Hu, S., Chen, M., Yang, C., Yang, B. X., ... \& Liu, Z. (2020). The mental health of medical workers in Wuhan, China dealing with the 2019 novel coronavirus. The Lancet Psychiatry.

Koh, D. (2020). Occupational risks for COVID-19 infection. Occupational medicine (Oxford, England), $70(1), 3$.

Leão, R. F., Oliveira, D. A., \& Aparecida, N. (2012). Entrevista-La salud del profesional y las condiciones de trabajo. Retratos da Escola, 6(11), $301-313$.

Mattos, J. G. S., Ferreira, W. L., Santana, L. C., de Souza Castro, S., \& Ferreira, L. A. (2022). Síndrome de Burnout em profissionais de saúde da linha de frente contra a Covid-19. Research, Society and Development, 11(1), e33211124923-e33211124923.

Moraes, É. B., Sanchez, M. C. O., Valente, G. S. C., de Souza, D. F., \& Nassar, P. R. B. (2020). A segurança dos profissionais de saúde em tempos de COVID-19: uma reflexão. Research, Society and Development, 9(7), e134973832-e134973832.

Nagesh, S., \& Chakraborty, S. (2020). Saving the frontline health workforce amidst the COVID-19 crisis: challenges and recommendations. Journal of global health, 10(1).

Prigol, A. C., \& Santos, E. L. (2020). Saúde mental dos profissionais de enfermagem diante da pandemia COVID-19. Research, Society and Development, 9(9), e542997563-e542997563.

Rache, B., Rocha, R., Nunes, L., Spinola, P., Malik, A. M., \& Massuda, A. (2020). Necessidades de Infraestrutura do SUS em Preparo ao COVID-19: Leitos de UTI, Respiradores e Ocupac ao Hospitalar.

Ramaci, T., Barattucci, M., Ledda, C., Rapisarda, V. (2020). Estigma social durante o COVID-19 e seu impacto nos resultados dos profissionais de saúde. Sustentabilidade. 12(9):3834

Tavares, V. (2020). Covid-19: a saúde dos que estão na linha de frente. Rio de Janeiro: Fiocruz. 